\title{
Physics-Guided Curve Fitting for Potential-Energy Functions of Diatomic Molecules
}

\author{
Karl Irikura ${ }^{1}$ \\ ${ }^{1}$ National Institute of Standards and Technology
}

April 22, 2021

\begin{abstract}
When computing the potential-energy curve of a diatomic molecule for predictive spectroscopy, high-level calculations are usually desired. The best calculations are expensive, so few points are usually available. The points are fitted to a continuous function, such as a polynomial. Ro-vibrational energy levels are then computed using the fitted function, and spectroscopic constants extracted. However, there may be problems with overfitting, with inadequate flexibility of the fitting function, or with dependence of results upon the choice of fitting function. More fundamentally, the fitting function is selected using aesthetics or convenience, instead of physics. Here we suggest using a lower-level, high-resolution ab initio potential as a guide. Instead of fitting the sparse, high-level data directly, the energy differences between the high-level points and the guiding potential are fitted. The results are improved even with an inexpensive guiding potential. This simple strategy involves little additional effort and can be recommended for routine use. It is similar to some interpolation strategies in the literature of polyatomic molecules. When the guiding potential extends beyond the high-level data, extrapolations are also improved.
\end{abstract}

\section{Hosted file}

guided_fit.pdf available at https://authorea.com/users/273269/articles/519061-physics-guidedcurve-fitting-for-potential-energy-functions-of-diatomic-molecules 